\title{
Situação epidemiológica da leishmaniose visceral, na Ilha de São Luís, Estado do Maranhão
}

\author{
Epidemiological situation of visceral leishmaniasis \\ on the Island of São Luis, State of Maranhão
}

\author{
Antonio Rafael da Silva ${ }^{1}$, Pedro Luiz Tauil ${ }^{2}$, Maria Neuza Souza Cavalcante ${ }^{1}$, \\ Maria Nilza Medeiros ${ }^{1}$, Benedito Nascimento Pires \\ e Eloísa da Graça do Rosário Gonçalves ${ }^{1}$
}

\begin{abstract}
RESUMO
Apresenta-se o resultado de um estudo de campo sobre a leishmaniose visceral autóctone da Ilha de São Luís. Com início em 2004 e término em 2006, a pesquisa visou conhecer aspectos epidemiológicos e clínicos determinantes da endemia. Foram analisados 299 casos autóctones, sendo 83,6\% em menores de 9 anos e 54,1\% do sexo masculino. 0 agravo ocorreu em todos os meses do ano com pico em junho. 0 coeficiente de incidência foi reduzido de 46,1 para 35,2 casos por 100.000 habitantes nos anos estudados. O diagnóstico teve confirmação laboratorial em 93,3\% dos casos. 0 tratamento de escolha foi à base de N-metilglucamina com percentual de cura de 96,1\%. A letalidade média foi de 3,7\%. Em função da inexistência de ações mais sistemáticas de controle, propõe-se a criação de um programa a ser desenvolvido pelos municípios sob a coordenação da Secretaria de Estado da Saúde.
\end{abstract}

Palavras-chaves: Leishmaniose visceral. Epidemiologia. Ilha de São Luís.

\section{ABSTRACT}

The results from a field study on autochthonous visceral leishmaniasis on the island of São Luís are presented. This study started in 2004 and finished in 2006 and had the aim of ascertaining the determinant epidemiological and clinical characteristics of this endemic disease. Two hundred ninety nine autochthonous cases were analyzed, of which $83.6 \%$ were children younger than 9 years old and $54.1 \%$ were male. The disease occurred in all months of the year, with a peak in June. The coefficient of incidence decreased from 46.1 to 35.2 cases per 100,000 inhabitants over the years studied. The diagnosis was confirmed by laboratory tests in $93.3 \%$ of the cases. The treatment of choice was based on N-methylglucamine, with a cure rate of $96.1 \%$. The mean lethality rate was $3.7 \%$. Because of the absence of systematic control actions, the authors propose the creation of a specific program to be developed by the municipalities under the coordination of the State Department of Health.

Key-words: Visceral leishmaniasis. Epidemiology. Island of São Luís.

A Organização Mundial da Saúde (OMS) reconhece a leishmaniose visceral (calazar) como um importante problema de saúde pública. Apesar de endêmica em mais de 60 países, a Índia, Bangladesh e Nepal (Ásia), o Sudão (África) e o Brasil (Américas), são responsáveis por cerca de $90 \%$ dos casos registrados no mundo $0^{51322}$.

A partir de 1950, com os trabalhos realizados no Ceará, Bahia e Piauí em humanos e animais, o calazar foi considerado endêmico no Brasil ${ }^{12}{ }^{12} 35$. Nas últimas duas décadas, a doença vem se expandindo numérica e geograficamente, incidindo atualmente em 19 das 27 unidades da federação, principalmente na Região Nordeste, responsável por $90 \%$ das notificações, particularmente nos Estados da Bahia, Ceará, Piauí e Maranhão ${ }^{1011253638}$.
A doença esteve ausente, no Maranhão, até o final da década de $1970^{6}$. Em 1980, começou a incidir em nosso meio ${ }^{32}$, a partir de bairros da Cidade de São Luís, logo se expandindo para outros municípios da Ilha de São Luís ${ }^{20} 253$. Em 1997, um dos autores, estudando a evolução do calazar na Ilha de São Luís, mostrou que a letalidade hospitalar anunciada, de 3,9\%, estava subestimada, sendo na realidade de $6,7 \%$, em decorrência dos óbitos que ocorreram nas residências de pacientes não notificados ${ }^{33}$. Em 2002, a equipe do Centro de Referência em Doenças Infecciosas e Parasitárias (CREDIP) da Universidade Federal do Maranhão (UFMA), levantando os dados de calazar encontrou uma letalidade de $11,7 \%$. Em 2003 , seus integrantes acompanharam as ocorrências de calazar e detectaram uma letalidade

\footnotetext{
1. Centro de Referência em Doenças Infecciosas e Parasitárias, Universidade Federal do Maranhão, São Luís, MA. 2. Núcleo de Medicina Tropical, Universidade de Brasília, Brasília, DF.

Endereço para correspondência: Dr. Antonio Rafael da Silva. Deptº de Patologia/UFMA. Praça Madre Deus 02, Térreo, 65025-560 São Luís, MA.

Telefax: $55983221-0270$

e-mail: regionalsbmt@elo.com.br

Recebido para publicação em 10/10/2007

Aceito em 01/07/2008
} 
de $12,5 \%$. Esses resultados foram apresentados e discutidos com os Secretários de Saúde dos municípios da Ilha e motivaram o desenvolvimento do presente trabalho em parceria, que teve como objetivos: estudar a situação epidemiológica do calazar na Ilha de São Luís; estudar os aspectos clínico, diagnóstico e terapêutico; conhecer a estrutura dos municípios, relacionada ao controle da doença.

\section{MATERIAL E MÉTODOS}

A Ilha de São Luís localiza-se no litoral norte do estado, na região do golfo maranhense. 0 clima é tropical úmido e a temperatura média varia entre $26^{\circ} \mathrm{C} \mathrm{e} 28^{\circ} \mathrm{C}$. A pluviometria anual atinge $2.000 \mathrm{~mm}^{3}$ e as chuvas são mais intensas de janeiro a junho. Inclui os municípios de São Luís, São José de Ribamar, Paço do Lumiar e Raposa. A Figura 1 e a Tabela 1 mostram as características geográficas e populacionais dos municípios.
A população, por grupo etário, segundo a Fundação Instituto Brasileiro de Geografia e Estatística, distribuiu-se em: 10,3\% entre 0 e 4 anos; $9,9 \%$ entre 5 e 9 anos; $24,3 \%$ entre 10 e 19 anos; $20,2 \%$ entre 20 e 29 anos; $14,7 \%$ entre 30 e 39 anos; $9,6 \%$ entre 40 e 49 anos; $5,3 \%$ entre 50 e 59 anos e 5,5\% em maiores de 60 anos.

0 trabalho teve início em janeiro de 2004 e término em dezembro de 2006. Contou com a participação de uma equipe formada por médicos e professores do Centro de Referência em Doenças Infecciosas e Parasitárias da Universidade Federal do Maranhão, agentes de saúde e técnicos das secretarias de saúde dos municípios envolvidos.

Após a notificação de um caso de calazar, a partir de dados do Sistema Nacional de Agravo e Notificação da Secretaria de Estado da Saúde (SINAN-MA), os seguintes procedimentos sucederam-se como fonte de dados para as análises: visita da equipe à localidade e entrevista com as famílias para investigação epidemiológica; identificação dos procedimentos laboratoriais empregados para a confirmação diagnóstica, a partir dos prontuários; visita à

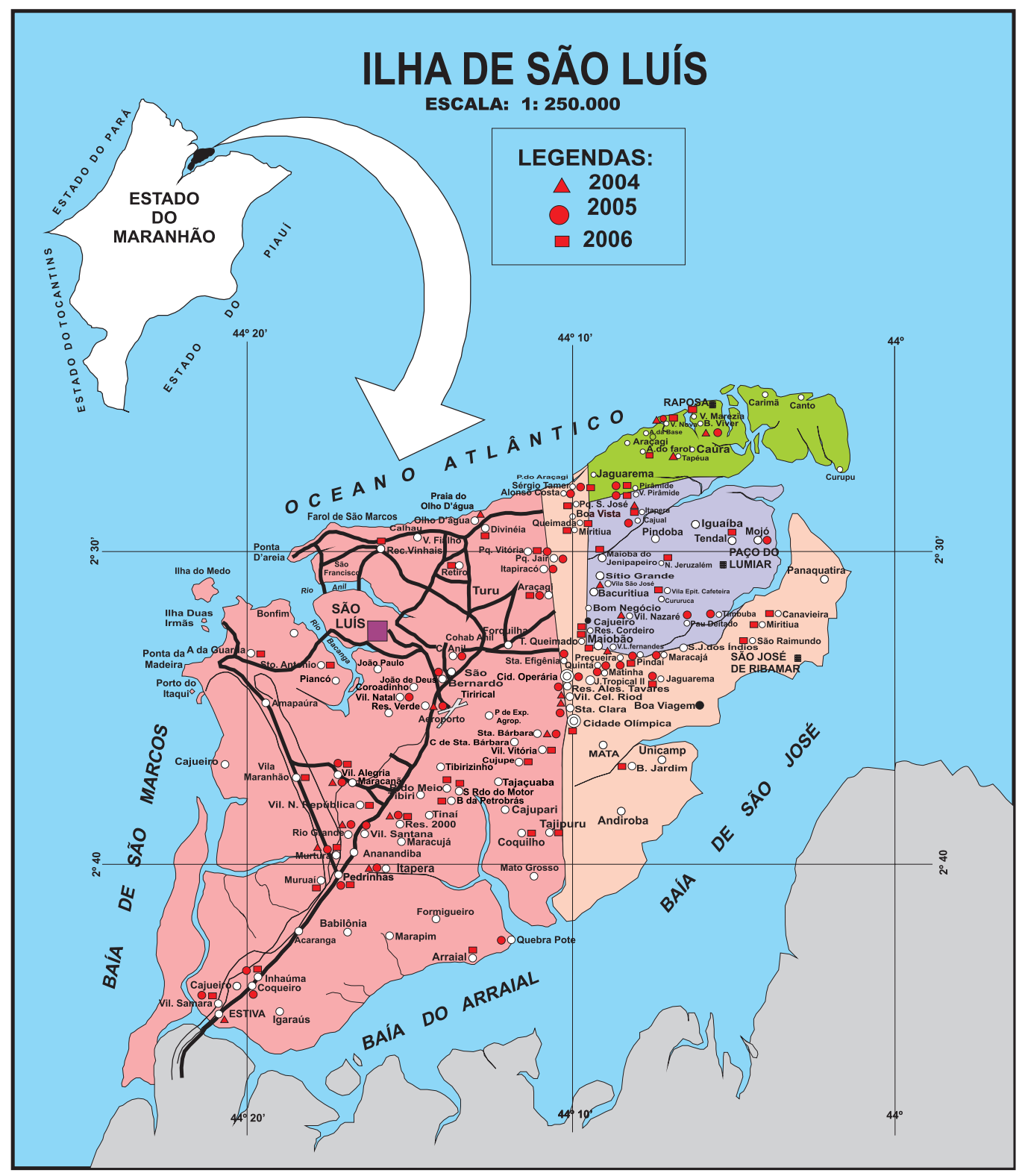

Figura 1- Localidades da Ilba de São Luís com casos de leishmaniose visceral americana, 2004 a 2006. 
Tabela 1 - Características geográficas e populacionais dos municípios da Ilba de São Luís, MA, 2004 a 2006.

\begin{tabular}{|c|c|c|c|c|c|c|}
\hline \multirow[b]{2}{*}{ Municípios } & \multirow[b]{2}{*}{ Área $\left(\mathrm{km}^{2}\right)$} & \multirow[b]{2}{*}{ Localidades } & \multicolumn{3}{|c|}{ População estimada } & \multirow[b]{2}{*}{$\mathrm{Hab} / \mathrm{km}^{2}$} \\
\hline & & & total & urbana & rural & \\
\hline São Luís & 827 & 338 & 978.777 & 871.111 & 107.666 & $1.183,5$ \\
\hline São José Ribamar & 386 & 103 & 130.437 & 65.479 & 64.958 & 337,9 \\
\hline Poço Lumiar & 132 & 73 & 97.679 & 43.662 & 54.017 & 740,0 \\
\hline Raposa & 64 & 42 & 20.713 & 15.327 & 5.386 & 323,6 \\
\hline Total & 1.410 & 556 & 1.227 .606 & 995.579 & 232.027 & 870,6 \\
\hline
\end{tabular}

unidade de saúde de referência do caso, entre 40 e 50 dias após o término do tratamento, para reavaliação clínica do paciente, adotando-se, para avaliação do tamanho do baço, a classificação de Hackett ${ }^{16}$; avaliação da organização das atividades de controle de endemias dos serviços municipais de saúde, especialmente em relação ao calazar.

Cada etapa do trabalho foi seguida de relatório circunstanciado e de avaliações com a equipe de acompanhamento, integrada por profissionais dos municípios e um representante do Conselho Municipal de Saúde. Os dados foram anotados na Ficha de Avaliação do Caso de Calazar e armazenados no programa EPIINFO 2002 para análises.

\section{RESULTADOS}

No decorrer do estudo foram registrados 428 casos de calazar, sendo 299 (69,8\%) autóctones da Ilha de São Luís. 0 local provável de infecção de 263 (88\%) destes pacientes foi na localidade de moradia e dos outros 36 (12\%), no próprio município, pois os pacientes não ultrapassaram os seus limites.

Houve 145 localidades acometidas: 68 no município de São Luís, com 151 casos; 43 no município de São José de Ribamar, com 68 casos; 19 no município de Paço do Lumiar, com 44 casos e 15 no município de Raposa, com 36 casos (Figura 1).

A curva anual de aparecimento da doença mostra que o calazar aconteceu em todos os meses do ano com acentuação do número de casos no mês de junho, final do período chuvoso (Figura 2).

A Tabela 2 apresenta os casos por grupo etário, registrandose ligeiro predomínio no sexo masculino $(54,2 \%)$. Naqueles com idade acima de 10 anos, a incidência nos homens foi aproximadamente duas vezes maior que nas mulheres. A taxa de letalidade geral foi de $3,7 \%$, representando uma redução de 70,4\% quando se compara aos 12,5\%, registrados em 2003.

A Tabela 3 mostra o coeficiente de incidência do calazar por município de risco, chamando-se a atenção para o seu alto valor no município da Raposa.

Os ambientes de origem dos pacientes, situam-se em terrenos com intensa cobertura vegetal, próximo a igarapés, regiões de praia e nascedouros de água. Guardam características nitidamente rurais e semi-rurais com as habitações localizadas em arruados ou mesmo isoladas, circundadas por grande variedade de plantações e árvores frutíferas. As habitações apresentavam condições físicas boas e regulares, com $56,8 \%$ delas cuidadosamente asseadas; A maioria $(67,4 \%)$ era coberta de telha de barro ou de amianto e as demais, cobertas de palha; as paredes, na maioria $(72,7 \%)$ eram tapadas de barro, sendo 69,4\% dos pisos de chão batido. Setenta e dois por cento das moradias eram próprias, $55 \%$ servidas por água de torneira, dentro ou fora da casa e nenhuma ligada à rede de esgoto. Os animais domésticos mais encontrados foram cão, gato, galinhas e, menos freqüentemente, suínos.

Cerca de $76,5 \%$ das famílias possuem de 4 a 7 membros e $20 \%$, entre 8 e 12 . A maioria dos chefes de família trabalha

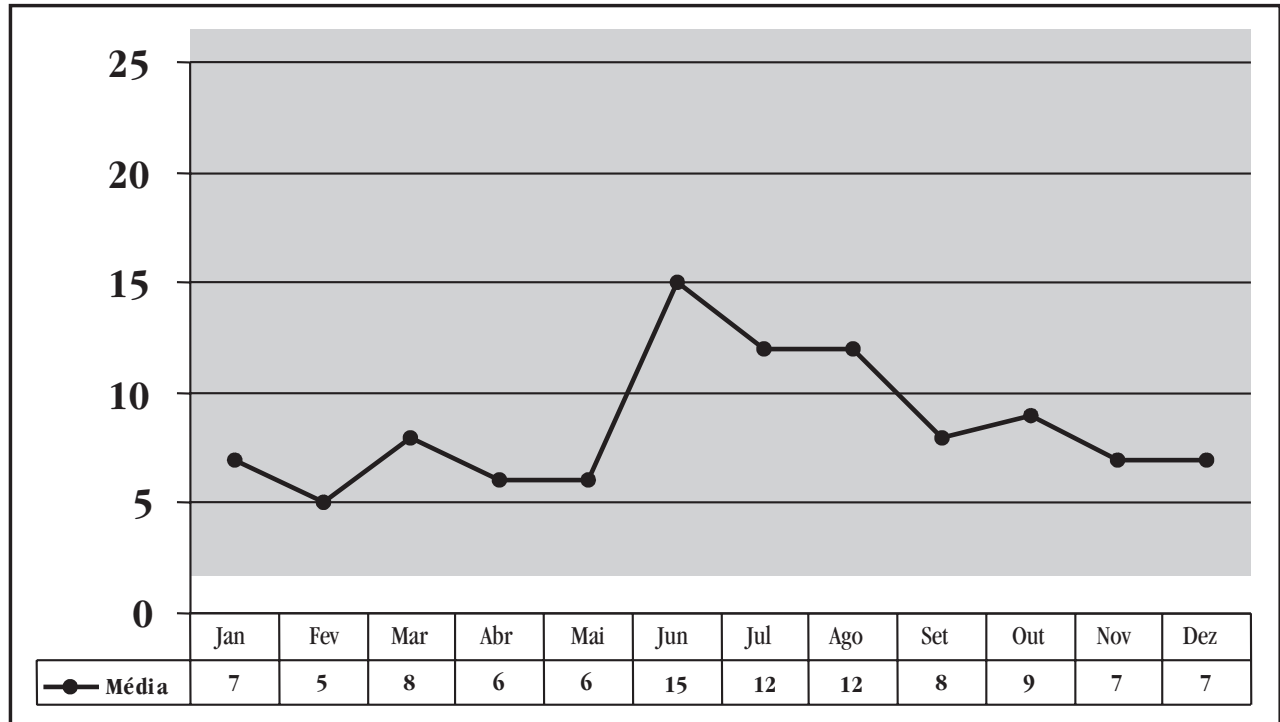

Figura 2 - Média dos casos de leishmaniose visceral (calazar) de janeiro a dezembro na Ilba de São Luís, MA, 2004 a 2006. 
Tabela 2 - Distribuição dos casos de calazar por grupo etário e sexo. Ilba de São Luís, MA, 2004 a 2006.

\begin{tabular}{|c|c|c|c|c|c|c|c|c|c|}
\hline \multirow[b]{2}{*}{ Grupo etário } & \multicolumn{2}{|c|}{ Masculino } & \multicolumn{2}{|c|}{ Feminino } & \multicolumn{2}{|c|}{ Total } & \multirow[b]{2}{*}{ Incidência* } & \multirow[b]{2}{*}{ Óbito } & \multirow[b]{2}{*}{ Let $^{* * *}$} \\
\hline & $\mathrm{n}^{0}$ & $\%$ & $\mathrm{n}^{0}$ & $\%$ & $\mathrm{n}^{0}$ & $\%$ & & & \\
\hline $0-23$ meses & 79 & 26,4 & 73 & 24,4 & 152 & 50,8 & - & 6 & 3,9 \\
\hline $2-4$ anos & 40 & 13,6 & 34 & & 74 & & $9,73^{* * * *}$ & 1 & 1,3 \\
\hline $5-9$ anos & 10 & 3,3 & 14 & 11,4 & 24 & 24,7 & 1,06 & - & - \\
\hline $10-19$ anos & 10 & 3,3 & 8 & 2.7 & 18 & 6,0 & 0,32 & - & - \\
\hline $20-29$ anos & 12 & 4,0 & 4 & 1.3 & 16 & 5,3 & 0,35 & 2 & 12,5 \\
\hline $30-39$ anos & 6 & 2,0 & 1 & 0,3 & 7 & 2,3 & 0,21 & - & - \\
\hline $40-49$ anos & 5 & 1,7 & 1 & 0,3 & 6 & 2,0 & 0,27 & - & - \\
\hline$>50$ anos & - & & 2 & 0,7 & 2 & 0,7 & 0,08 & 2 & 12,5 \\
\hline Total & 162 & 54,2 & 137 & 45,8 & 299 & 100,0 & 0,43 & 11 & 3,7 \\
\hline
\end{tabular}

Tabela 3 - Coeficiente de incidência do calazar por município, Ilba de São Luís, MA, 2004 a 2006.

\begin{tabular}{|c|c|c|c|c|c|c|c|c|c|c|}
\hline \multirow[t]{2}{*}{ Ano } & \multicolumn{2}{|c|}{ São Luís } & \multicolumn{2}{|c|}{ São José Ribamar } & \multicolumn{2}{|c|}{ Paço do Lumiar } & \multicolumn{2}{|c|}{ Raposa } & \multicolumn{2}{|c|}{ Total/Ilha } \\
\hline & casos & $\mathrm{CI}^{*}$ & casos & $\mathrm{CI}^{*}$ & casos & $\mathrm{CI}^{*}$ & casos & $\mathrm{CI}^{*}$ & casos & $\mathrm{CI}^{*}$ \\
\hline 2004 & 58 & 54,9 & 18 & 28,6 & 16 & 30,8 & 12 & 229,7 & 104 & 46,1 \\
\hline 2005 & 60 & 55,7 & 25 & 38,4 & 18 & 23,3 & 7 & 130,0 & 110 & 46,9 \\
\hline 2006 & 33 & 30,6 & 25 & 37,3 & 10 & 17,8 & 17 & 306,3 & 85 & 35,2 \\
\hline Total & 151 & 68 & 44 & 36 & 299 & & & & & \\
\hline
\end{tabular}

informalmente em atividades referidas como biscateiro, braçal, pescador, pedreiro, lavrador e vigilante. Outras ocupações foram carroceiro, lanterneiro, cozinheiro, motorista, encadernador, fiscal, cobrador, balconista, servente e vaqueiro. Cerca de 15\% declararam-se aposentados ou em vias de aposentadoria. Quanto às mulheres, 72\% declararam-se domésticas, ajudando na economia do lar pelo cultivo de hortas. Outras emprestam sua mão-de-obra em atividades de lavadeira, biscateira, faxineira, lavradora e professora.

A percepção do calazar na família dos acometidos era de preocupação. Somente $10 \%$ mostraram-se tranquiilas, alegando saberem que a doença tinha cura e que a medicação específica era gratuita. No aspecto financeiro foi freqüente ouvir das famílias que $o$ calazar é um desassossego, pois foram obrigadas a levar o filho várias vezes aos serviços de Saúde e a gastar com a compra de remédios. Após longa espera, a internação e com esta, outro transtorno familiar: pelo menos uma pessoa parava de trabalhar para tomar conta da família, enquanto outra acompanhava o paciente no hospital.

Foi apurado nas entrevistas que 55\% das famílias tinham história de calazar atual ou anterior. Por essa razão, conheciam o seu modo de transmissão, ligando-o à existência de cães doentes e de insetos; neste ponto, queixaram-se do pessoal da Saúde que dá pouca importância ao fato. Paradoxalmente, $85 \%$ das famílias possuíam cão e, mesmo que o animal estivesse com aspecto doentio, recusavam o seu recolhimento indicado por profissionais de Saúde.

A procura por atendimento nas unidades de Saúde ocorreu, em 45\% das famílias, entre 5 e 40 dias do início dos sintomas; neste grupo estavam aqueles cuja febre era, desde o início, contínua e alta. Outros $35 \%$ preferiram utilizar, inicialmente, remédios caseiros, incluindo garrafadas e $20 \%$ ficaram na expectativa de melhora espontânea do quadro por acharem que não era nada. Era explicado, neste caso, que o retardo em procurar auxílio médico devia-se à irregularidade da febre, que cedia com antitérmicos, ou por residirem em lugares distantes dos serviços de Saúde.
Antes de terem a confirmação diagnóstica do calazar, 20\% dos pacientes foram internados, segundo os familiares, devido à pneumonia bacteriana, dengue, virose, esquistossomose, desnutrição e malária. Não houve relato de que o médico tenha realizado exame físico como critério para internação, nem de que a presença de emagrecimento, palidez, aumento do volume abdominal e a longa duração da doença levaram à suspeita clínica de calazar naqueles pacientes.

A Tabela 4 refere as manifestações clínicas que orientaram a procura de atenção médica sendo as mais frequientes: febre, palidez, emagrecimento e protusão abdominal, embora os mais preocupantes tenham sido tosse, falta de ar, vômitos e diarréia quando acompanhados de febre.

Cento e trinta $(43,5 \%)$ pacientes realizaram até 3 consultas, $99(33,1 \%)$ pacientes 4 ou 5 e $51(17 \%)$, mais de 5 consultas, antes de terem o diagnóstico confirmado. Outros 19 (6,3\%) não lembraram de quantas vezes procuraram os ambulatórios. Quando a Unidade de Saúde estava próxima à residência, 80\% referiram facilidade de atendimento, a grande maioria respondendo que encontrava 0 médico e os enfermeiros. No entanto, quando questionados sobre a qualidade das consultas, 55\% dos entrevistados afirmaram que o médico somente olhava, receitava e não examinava o quê, para eles, contribuía para as idas e vindas aos Serviços.

Em 20 (6,7\%) pacientes os sintomas tiveram início no ano anterior à pesquisa ( 5 no primeiro e 15 no segundo semestre). 0 intervalo médio entre o início dos sintomas e o início do tratamento na Ilha foi de 66,6 dias, sendo em: São Luís, 48,3 dias; Paço do Lumiar, 58,2 dias; São José de Ribamar, 75 dias; e Raposa 85 dias.

A Figura 3 mostra os resultados dos exames laboratoriais utilizados para confirmação do diagnóstico de calazar, todos realizados no Hospital Universitário e no Centro de Referência em Doenças Infecciosas e Parasitárias. A maioria (63,2\%) dos pacientes teve o diagnóstico confirmado pela demonstração 
Tabela 4 - Sintomas referidos por 260 pacientes de calazar, Ilba de São Luís, MA, 2004 a 2006.

\begin{tabular}{lcc}
\hline Sintomatologia & Frequiência & Percentual \\
\hline Febre & 254 & 96,1 \\
Palidez & 247 & 95,0 \\
Emagrecimento & 241 & 92,6 \\
Protusão abdominal & 217 & 83,4 \\
Tosse & 152 & 58,4 \\
Calafrios & 130 & 50,0 \\
Vômitos & 103 & 39,6 \\
Diarréia & 105 & 40,3 \\
Alteração da cor da pele & 102 & 39,2 \\
Dores de cabeça & 72 & 27,0 \\
Falta de ar & 42 & 16,0 \\
Edema de membro inferior & 37 & 14,2 \\
Queda de cabelo & 32 & 12,3 \\
\hline
\end{tabular}

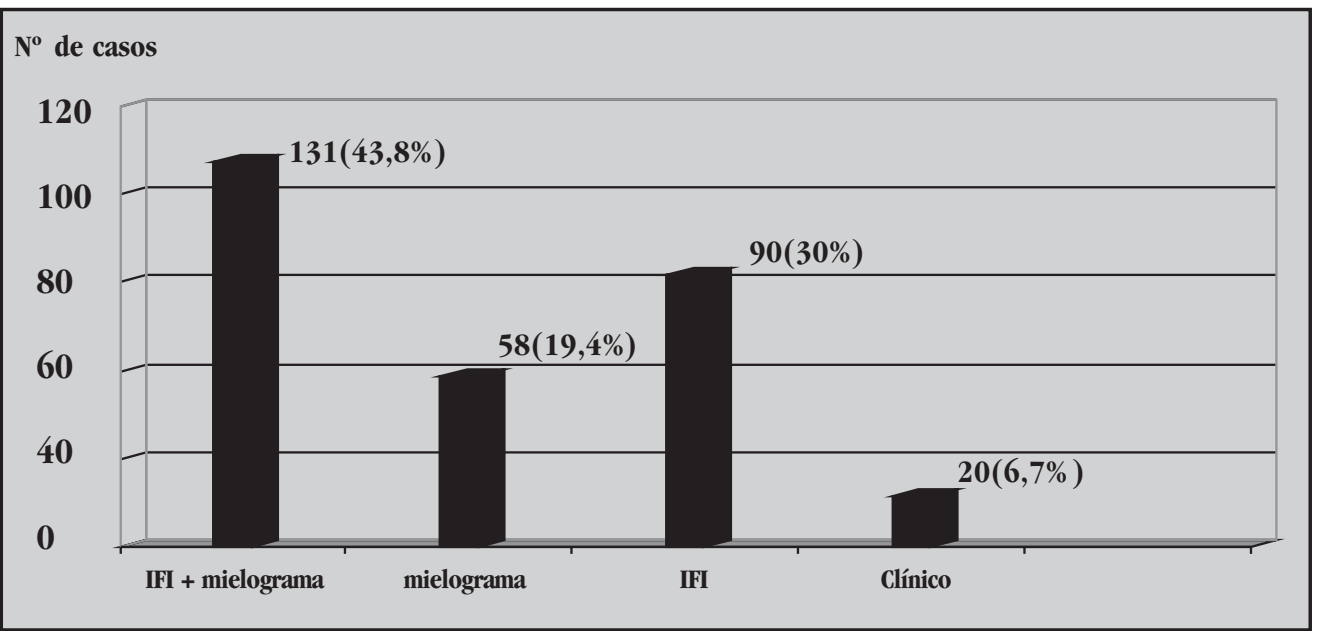

Figura 3 - Percentual de casos de leishmaniose visceral (calazar) segundo critério de confirmação do diagnóstico. Ilba de São Luís, MA, 2004 a 2006.

do parasita no aspirado da medula óssea. A reação de imunofluorescência indireta (IFI) foi realizada em 221 pacientes, sendo que em 75,5\% deles os anticorpos apresentavam títulos iguais ou superiores a 1/160. Em 20 pacientes o diagnóstico se baseou apenas em dados clínicos e epidemiológicos, estando todos com menos de 30 dias de doença. Destes, 5 realizaram IFI e punção medular, com ambos os resultados negativos; 6 realizaram apenas IFI com resultado não reagente e 9 não foram submetidos a exames confirmatórios.

Nas visitas de campo para revisão dos pacientes após o término do tratamento, foi possível realizar exame físico em 165 deles, constatando-se estado geral regular ou bom, com mucosas hipocoradas em 32 e ausência de sinais esteto-acústicos anormais; 6 apresentavam temperatura entre $37,5^{\circ} \mathrm{C}$ a $38,5^{\circ} \mathrm{C}$; à palpação abdominal presença de fígado e baço palpáveis, em 113 (68,5\%) pacientes. Entre estes, 78 (69\%) apresentavam baço, grau I, 19 (16,8\%), grau II, 12 (10,6\%), grau III e 4 (3,5\%), grau IV. Todos os pacientes sintomáticos foram selecionados para seguimento, apresentando regressão do quadro nos meses seguintes.

0 tratamento de $92,6 \%$ dos pacientes ocorreu nas unidades do Sistema Único de Saúde (SUS), do município de São Luís.
Trinta e cinco (12,7\%) diagnosticados no Centro de Referência em Doenças Infecciosas e Parasitárias foram encaminhados para tratamento nas unidades de Saúde dos seus municípios. Estes pacientes foram acompanhados com Ficha de Acompanhamento de Tratamento e revistos com os profissionais das referidas unidades de Saúde até a alta definitiva. Dentre eles, 3 apresentavam co-infecção (dois com aids e um com esquistossomose).

Em todas as unidades, a droga utilizada para tratamento dos pacientes foi a $\mathrm{N}$-metil glucamina (glucantime), na dose de $20 \mathrm{mg} / \mathrm{Sb}^{\mathrm{V}} / \mathrm{kg} / \mathrm{peso} / \mathrm{dia}$, aplicados durante 20 dias, em injeção endovenosa ou intramuscular, de acordo com as normas do Ministério da Saúde ${ }^{22}$. A eficácia da medicação neste primeiro curso de tratamento foi de $93,2 \%$. Houve 12 pacientes resistentes ao tratamento, sendo cinco co-infectados com HIV. Estes 12 pacientes foram retratados com anfotericina-B, com boa resposta clínica.

Para conhecer a participação dos Serviços no controle do calazar, além das visitas locais, realizaram-se reuniões de avaliação que permitiram identificar a inexistência de um plano de trabalho que permitisse atuações sustentáveis. Falta de verbas, de veículos, de pessoal e de insumos, era sistematicamente aventada. 0 Centro 
de Controle de Zoonoses do Município de São Luís e o Laboratório Central (LACEN) realizaram as sorologias caninas, o primeiro tendo sido responsável pela eliminação dos 5,1\% de cães com sorologia positiva. Os dados disponíveis sobre cães mostram que, no período de estudo, a Ilha de São Luís tinha em registro um número variável entre 160 e 165 mil cães. 0 recolhimento e a eliminação são feitos de maneira descontínua: entre cães com sorologia positiva e aqueles com solicitação para recolhimento, somente $40 \%$ chegaram a ser eliminados. Igual situação ocorre em relação ao controle de vetores.

Sobre a rede de serviços de atendimento aos pacientes (postos, centros de saúde e hospitais), o Município de São Luís adota o sistema de distritalização. Os demais municípios fizeram tentativas para sua criação, mas sem sucesso, embora tenham aumentado a rede de serviços nos três anos estudados, suficiente para cobrir a demanda de atendimentos. 0 número de servidores é satisfatório para atender a demanda dos municípios, porém 90\% das famílias afirmaram que somente os agentes da FUNASA visitavam periodicamente as suas localidades. O Programa de Saúde da Família (PSF) vem se estruturando e expandindo a cada ano, embora tenha sido praticamente inexistente sua participação no diagnóstico e tratamento do calazar.

\section{DISCUSSÃO}

A Ilha de São Luís se inclui entre as capitais e outros municípios brasileiros onde a leishmaniose visceral passou a ser uma endemia urbana ${ }^{18}$. Assim, constitui uma referência de urbanização do calazar no Brasil, a partir de 1980, com a instalação do Complexo Industrial do Maranhão, que obrigou milhares de famílias a se instalarem em outras áreas em condições inadequadas de habitação e saneamento ${ }^{31}$. Um dos primeiros reflexos desse desequilíbrio sócio-ambiental foi o surto de calazar ocorrido em 1982 com registro de 32 casos da doença em dois bairros periféricos do município de São Luís ${ }^{32}$.

Os altos coeficientes de letalidade levaram os autores a estudar a situação atual da endemia para conhecer sua expansão geográfica e compreender a dinâmica de transmissão. Moreira Jr e cols ${ }^{24}$, em diferentes bairros da Cidade de Jequié-BA, definidos como de alto e baixo risco, concluíram que a discrepância entre a incidência de LVH nos bairros estudados sugere que a ruralização da periferia da cidade favorece a ocorrência de LVH e não que o calazar esteja se urbanizando. Esse processo parece estar ocorrendo na Ilha de São Luís. A expansão de endemias transmitidas por vetores atinge populações que, num esforço de sobrevivência, migram, promovem modificações ambientais, submetem-se a condições de insalubridade, sendo esses elementos epidemiológicos de vulnerabilidade (circulação do parasita), receptividade (existência de vetores) e suscetibilidade (população sujeita ao risco), facilitadores da instalação de doenças ${ }^{32030}$. Um bom exemplo é o calazar ${ }^{142031323437}$.

0 presente trabalho permitiu aos autores observar a grande dispersão do calazar, alta incidência, a continuidade do precário modo devida das populações e a continuidade das ocupações/invasões na llha de São Luís, tudo contribuindo para a expansão da endemia ${ }^{20} 33$.
À semelhança de outros estudos ${ }^{702136}$, ocorre em todos os meses do ano, independente das alterações metereológicas, o que dificulta o estabelecimento do momento mais apropriado para executar uma das intervenções de controle anti-vetorial, a borrifação, que é pouco ou irregularmente praticada.

Sabe-se que, de regra, o calazar atinge, preferencialmente, a população infantil ${ }^{1821} 2628$. Neste estudo, 83,6\% dos casos aconteceram em menores de 9 anos, fato epidemiologicamente indicador da proximidade precoce do ser humano com o cão, reservatório da infecção e com o vetor da doença em nosso meio (Lutzomyia longipalpis) $^{425}{ }^{29}$. As manifestações encontradas mostram que não há grandes dificuldades técnicas para 0 diagnóstico, salvo aqueles casos em fase inicial, pois a clínica do calazar traduz-se por febre arrastada, quase sempre acompanhada de palidez, emagrecimento e hepatoesplenomegalia, associadas à pancitopenia e à hipergamaglobulinemia ${ }^{19} 39$.

Estudos comprovam que o controle do calazar baseado no diagnóstico precoce ainda, lamentavelmente, não se estabeleceu. Os altos percentuais encontrados de palidez, emagrecimento, edema, queda de cabelos e protusão abdominal por hipertrofia de baço e de fígado, revelam o longo tempo decorrido entre os primeiros sintomas e o diagnóstico. Em nosso meio este tempo está em torno de 60 dias, praticamente igual aos encontrado em Mato Grosso ${ }^{21}$, exprimindo, baixa qualidade da atenção à saúde. Apesar destes dados, a letalidade de 3,7\% observada ao final do estudo, é bem menor que as encontradas nos estados de São Paulo, Piauí, Minas Gerais e Mato Grosso ${ }^{71521}$; houve ainda melhora na confirmação do diagnóstico e, embora pequena, redução da incidência da doença na Ilha de São Luís.

É possível que estes resultados decorram das ações desenvolvidas na parceria com os municípios, apesar do estudo ter revelado a inexistência de ações mais sistemáticas de controle por parte destes, e, por essa razão, concorda-se com outros autores de que até o momento, a endemia pouco se beneficiou das clássicas estratégias de controle indicadas, mas não realizadas ${ }^{782326}$ o que em parte pode decorrer dos baixos indicadores sociais dos municípios em foc $0^{27}$ com capacidade reduzida de investimentos em ações integradas de controle.

Frente as dificuldades e a própria realidade dos municípios em manter a sustentabilidade dos seus programas, propõe-se um programa de controle integrado das doenças transmitidas por vetores a ser desenvolvido pelos municípios sob a coordenação da Secretaria de Estado da Saúde, retomando as idéias preconizadas por Leavell e Clark ${ }^{17}$, envolvendo: a) ações de prevenção primária (desenvolver atividades de educação ambiental e em saúde que promovam, por parte das comunidades afetadas, atitudes de fortalecimento do controle do vetor e dos reservatórios) e ações de prevenção secundária (trabalhar os sistemas locais de saúde, objetivando o diagnóstico precoce e o tratamento imediato dos pacientes; b) os municípios a por em prática as medidas preconizadas no programa de controle da LVA do Ministério da Saúde; c) ações de controle que agreguem o ente acometido, o ambiente de ocorrência do agravo, a organização dos serviços, a participação da comunidade, a atenção básica e a educação em Saúde; d) treinamento para profissionais da saúde. 


\section{REFERÊNCIAS}

1. Alencar JE. Expansão do Calazar no Brasil. Ceará Médico, 5:86-102, 1961.

2. Aragão, TC. Surto de Leishmaniose Visceral na Zona Norte do Ceará. In: $3^{\text {a }}$ Reunião de Saúde Pública do Estado do Ceará. Ceará,1953

3. Barreto MP. Movimento migratório e sua importância em epidemiologia. Revista da Sociedade Brasileira de Medicina Tropical 1:91-102, 1967

4. Barros VLL. Estudo da importância do Gallus gallus como chamariz de Lutzomyia longipalpis (Díptera, Psychodidae, Phlebotominae) no ambiente doméstico: caracterização de fonte alimentar do flebotomíneo na localidade de Preiçueira, município de São José de Ribamar - MA, no ano de 2005. Dissertação de Mestrado, Universidade Federal do Maranhão, São Luis, MA, 2006.

5. Bern C; Joshi AB, Lal Das M, High Tower, GD, Thakun GO, Bista MD. Factors associated with visceral leishmaniasis in Nepa: bed-net use is strongly perspective. American Journal of Tropical Medicine and Hygiene 63: 184-188, 2000.

6. Brandão AAR. Leishmaniose visceral no Maranhão. Relato de Caso. Revista da Sociedade de Parasitologia e Doenças Tropicais do Maranhão 20:156-157, 1974.

7. Camargo-Neves ULF, Spindola R, Lage L. A Leishmaniose visceral americana no Estado de São Paulo. Situação Epidemiológica em 2001-2002. Revista da Sociedade Brasileira de Medicina Tropical volume 36 (supl II): 27-29, 2003.

8. Carmo EH. Leishmaniose visceral no Brasil: situação atual, principais aspectos epidemiológicos, clínicos e medidas de controle. Revista da Sociedade Brasileira de Medicina Tropical 35 (supl III): 41, 2002.

9. Costa DL; Costa CHN. Mortalidade por Leishmaniose visceral em Teresina, Piauí. Revista da Sociedade Brasileira de Medicina Tropical 37 (supl I): 240-241, 2004.

10. Costa JML, Viana GMC, Saldanha ACR, Nascimento MDSB, Alvim AC, Burattini MN, Silva AR. Leishmaniose visceral no Estado do Maranhão, Brasil: a evolução de uma epidemia. Cadernos de Saúde Pública 11:321-324,1995.

11. Costa CHN, Pereira HF, Araújo MV. Epidemia de leishmaniose visceral no Estado do Piauí, Brasil (1980-1986\}. Revista de Saúde Pública 24:361-372, 1990.

12. Deane LM, Deane MP. Visceral leishmaniasis in Brasil: geografical distribuition and transmission. Revista do Instituto de Medicina Tropical de São Paulo 4:198212, 1962.

13. Desjeux P. Leishmaniasis: current situation and new perspectives. Comparative Imunology, Microbiology and Infectious Diseases 27:305-318, 2004.

14. Di Lourenzo C, Proietti FR, Assunção RM. Urbanização da leishmaniose visceral no Brasil - uma breve revisão. Revista da Sociedade Brasileira de Medicina Tropical 33 (supl I):316-317, 2000.

15. Filogônio LBS, Rabello A, Luz ZMP. Fatores associados à evolução clínica e à letalidade da leishmaniose visceral em pacientes atendidos em Belo Horizonte In: Resumos de XXI Reunião de Pesquisa Aplicada em Doenças de Chagas e Leishmaniose, Uberaba, 2005

16. Hackett LW. Spleen measurament in malaria. Journal National Malaria Society 3:121-33, 1944.

17. Leavell H, Clark EG. Preventive medicine for de doctor in his community: an epidemiological approach. Mac.Graw Hill, New York, 1965.

18. Marzochi MCA, Marzochi KBF. Leishmanioses em áreas urbanas. Revista da Sociedade Brasileira de Medicina Tropical 30 (supl I):162-164,1997.

19. Mayrink W, Magalhães PA. Diagnóstico de calazar. Revista do Instituto de Medicina Tropical de São Paulo 11:11-12, 1969

20. Mendes WS, Trovão JR, Silva AAM, Silva AR, Costa JMC. Expansão da leishmaniose visceral americana em São Luis, Maranhão, Brasil. Revista da Sociedade Brasileira de Medicina Tropical 35:227-231, 2002
21. Mestre GLC, Fontes CJF. A Expansão da epidemia de leishnamiose visceral no Estado de Mato Grosso, 1992-2005. Revista da Sociedade Brasileira de Medicina Tropical 40: 42-48, 2007.

22. Ministério da Saúde. Secretaria de Vigilância em Saúde. Manual de Vigilância e Controle da Leishmaniose Visceral. Secretaria de Vigilância em Saúde. Ministério da Saúde, Brasília, 2003

23. Monteiro OS. Leishmaniose visceral no Brasil. Perspectiva de controle. Revista da Sociedade Brasileira de Medicina Tropical 35 (supl I): 335, 2002.

24. Moreira Jr ED, Torres EB, Lobo CFL. Urbanização do calazar ou ruralização da periferia dos centros urbanos? Revista da Sociedade Brasileira de Medicina Tropical 33 (supl II): 100, 2006.

25. Nascimento MDSB, Fiori BIP, Carneiro LS, Burattini MN. Estado atual da leishmaniose visceral no Maranhão: aspectos epidemiológicos determinantes na manutenção da leishmaniose visceral no Estado do Maranhão, Brasil. Revista da Sociedade Brasileira de Medicina Tropical 29:233-240, 1996.

26. Oliveira ALL, Paniago AMM, Dorval MEC, Oshiro ET, Leal CR, Sanches M, Cunha RV, Bóia MR. Foco Emergente de leishmaniose visceral em Mato Grosso do Sul. Revista da Sociedade Brasileira de Medicina Tropical 39: 446-450, 2006

27. Pochman M, Amorim R (org) Atlas da exclusão no Brasil. $2^{\mathrm{a}}$ edição, Editora Cortez, São Paulo, 2003.

28. Queiroz MJA, Alves JGB, Correia JB. Leishmaniose visceral: características clínico-epidemiológicas em crianças de área endêmica. Jornal de Pediatria 80:141-146, 2004.

29. Rebelo JMM, Araújo JAC, Carvalho VLLB, Silva FS e Oliveira ST. Flébotomos (Díptera, Phlebotominae) da Ilha de São Luis, zona do Golfão Maranhense, Brasil. Revista da Sociedade Brasileira de Medicina Tropical 32:247-253, 1999.

30. Sabroza PC, Toledo LM, Osanai CH. A organização do espaço e os processos endêmico-epidêmico. In: Saúde, ambiente e desenvolvimento: processo e consequiências sobre as condições de vida 2:57-77, 1992.

31. Silva, AR. Malária: Fotografia de uma crise no setor Saúde. Editora da Universiddae Federal do Maranhão, São Luis, 1989.

32. Silva AR, Costa JML, Mochel A, Carneiro EWB, Brasil R. Leishmaniose visceral na Ilha de São Luis, Estado do Maranhão. I. Aspectos clínicos e terapêuticos. In: Resumos do XIX Congresso da Sociedade Brasileira de Medicina Tropical, Rio de Janeiro, p.65, 1983

33. Silva AR, Viana, GMC, Varonil C, Pires B, Nascimento MDSD, Costa JML. Leishmaniose visceral (calazar) na Ilha de São Luis, Maranhão, Brasil: evolução e perspectivas. Revista da Sociedade Brasileira de Medicina Tropical 30: 359-368, 1997.

34. Silva ES, Gontijo CMF, Pacheco RS, Fiúza VOP, Brasil RP. Visceral leishmaniasis in the metropolitan region of Belo Horizonte, State of Minas Gerais, Brasil. Memórias do Instituto Oswaldo Cruz 96:205-291, 2001

35. Silva JR. Leishmaniose visceral (calazar). Thesis. Faculdade Nacional de Medicina, Universidade do Brasil, Rio de Janeiro, 1957.

36. Simplício ACR, Furtado JBV, Monteiro OS. Leishmaniose visceral no Brasil. Análise epidemiológica nos últimos 16 anos. Revista da Sociedade Brasileira de Medicina Tropical 33(supl I): 298, 2000

37. Tavares LMSA. Avaliação do processo de urbanização da leishmaniose viscera no município de Aracaju, Sergipe. Revista da Sociedade Brasileira de Medicina Tropical 33 (supl I): 37, 2000

38. Viana GM, Costa JML, Silva AR. Leishmaniose visceral no Maranhão 1960 a 1983 Revista da Sociedade Brasileira de Medicina Tropical 27 (supl I): 240, 1994.

39. World Health Organization. Control of leishmaniasis. Expert Commit, 1995 